Röntgendosen immerhin in $4,1 \%$ der beobachteten Anaphasezellen. Dieses Verhalten ist nicht durch einen systematischen Unterschied in der Dosismessung bei Röntgen- und Elektronenstrahlen zu erklären. Ein solcher in der Größenordnung von $10 \%$ durchaus möglicher Effekt müßte aber die Elektronen- und Röntgenkurven in allen Fällen durch die gleiche Anderung des Abszissenmaßstabes zur Dekkung bringen lassen.
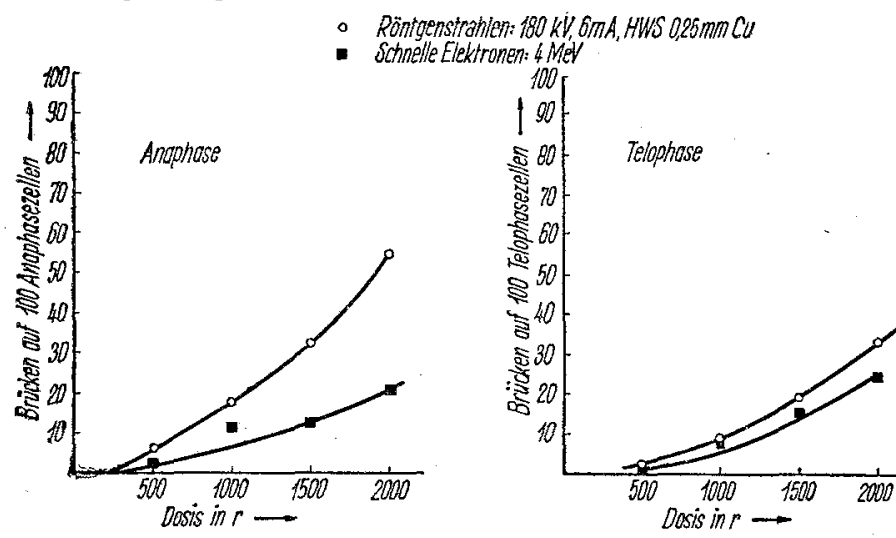

Fig.. 2 Häufigkeit von Chromosonenbrücken in Wurzelspitzenzellen der Gerste bei Bestrahlung mit Röntgenstrahlen vor schnellen Elektronen.

Nach den vorliegenden Ergebnissen nehmen die Wirkungskurven der Röntgenstrahlen und schnellen Elektronen den gleichen Verlauf, es ändert sich lediglich das Ausmaß der Wirkung. In der relativ geringen Wirkung kleiner Dosen von schnellen Elektronen scheint ein wesentlicher Unterschied im Verhalten der beiden Strahlungen bei der Auslösung von Chromosomenbrüchen zu liegen. Hinsichtlich der dafür verantwortlichen Trefferereignisse läßt sich aus den Dosiseffektkurven ableiten, daß das Zustandekommen des jeweiligen strahlenbiologischen Effekts sicherlich durch mehr als einen Treffer bedingt ist.

Strahlenbiologische Abteilung der Universitäts-

Frauenklinik (Dir.: Prof. Dr. H. Martius) und II. Physikalisches Institut der Universität Göttingen.

H. Bleek, W. Paul,

H. J. Schmermund und G. Schubert.

Eingegangen am 8. Januar 1949.

1) Die Untersuchungen wurden mit Unterstützung der LeibnizStiftung für Kunst und Wissenschaft durchgeführt. Prof. Dr. L angend orff, Freiburg, und Dr. Kaplan, Voldagsen, sind wir für wertvolle Ratschläge zu Dank verpflichtet.

\section{Untersuchungen über die tumorhemmende Wirkung des Miraeils.}

Im wissenschaftlich-chemischen Laboratorium der Bayer-Forschungsstätten in Elberfeld wurden 1938 von $\mathrm{H}$. Ma u $\beta^{1}$ ) básisch alkylierte Aminoxanthone synthetisiert, von welchen besonders die Verbindung Mis 752 mit der Formel

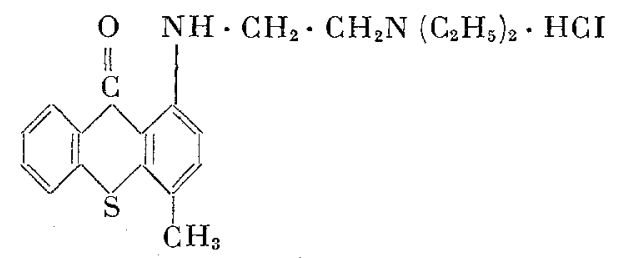

unter der Bezeichnung Miracil D von Kikuth ${ }^{2}$ ) und Gönnert im Bilharziosetest bei Affe und Maus als wirksam gefunden wurde.

Bei Untersuchungen über den Wirkungsmechanismus dieses Präparates fand $R$. Gönnert ${ }^{3}$, da $\beta$ das Miracil im Hoden, Ovar und Dotterstock der Bilharzien die Kernteilung verhindert oder zumindest stark hemmt, und'zwar seheinen bereits im Gange befindliche Kernteilungen ungestört abzulaufen, doch bleiben weitere Mitosen aus. $R$. Weyland $d^{4}$ konnte bei Untersuchungen an der Alliumwurzel die Mitosehemmung bestätigen.

Nach diesen Beobachtungen war es interessant festzustellen, ob dem Miracil und ähnlichen Verbindungen eine tumorhemmende Wirkung zukommt.

Die im Institut für experimentelle Pathologie der Bayer-Forschungsstatten von $\mathrm{G}$. Hackmann in dieser Richtung angestellten Versuche ließen beim W a l lk e r carcinom der Ratte bei bestimmter Dosierung deutliche Hemmungswirkungen, erkennen, während beim Ehrlichcarcinom ein Einfluß nicht deutlich nachzuweisen war.

I. Ehrlic h carcinom der Maus, intramusculär geimpft.

\begin{tabular}{|c|c|c|c|c|c|c|}
\hline Dosis $\mathrm{mg} / \mathrm{kg}$ & Zahl & $\dagger$ & neg. & pos. & $\begin{array}{l}\theta \text { Tumor } \\
\text { gewicht }\end{array}$ & Bemerkungen \\
\hline $\begin{array}{l}\text { 1. Versuch: } \\
\text { unbehandelt } \\
\text { Miracil sc. } \\
6 \times 100\end{array}$ & $\begin{array}{l}10 \\
10\end{array}$ & $\frac{2}{7}$ & $=$ & $\begin{array}{l}8 \\
3\end{array}$ & $\begin{array}{l}0,50 \mathrm{~g} \\
0,33 \mathrm{~g}\end{array}$ & $\begin{array}{l}\text { toxische } \\
\text { Wirkung }\end{array}$ \\
\hline $\begin{array}{l}\text { 2. Yersuch: } \\
\text { unbehandelt } \\
\text { Miracil sc. } \\
9 \times 50\end{array}$ & $\begin{array}{l}10 \\
10\end{array}$ & $\begin{array}{l}3 \\
3\end{array}$ & $=$ & $\begin{array}{l}7 \\
7\end{array}$ & $\begin{array}{l}0,64 \mathrm{~g} \\
0,57 \mathrm{~g}\end{array}$ & \\
\hline
\end{tabular}

II. W a lk er carcinom der Ratte, subcutan geimpft.

\begin{tabular}{|c|c|c|c|c|c|c|}
\hline $\begin{array}{l}\text { 3. Versuch: } \\
\text { unbehuandelt } \\
\text { Miracil D } \\
6 \times 100 \mathrm{sc} \text {. }\end{array}$ & $\begin{array}{l}14 \\
14\end{array}$ & $\overline{4}$ & $\begin{array}{l}1 \\
2\end{array}$ & $\begin{array}{r}18 \\
8\end{array}$ & $\begin{array}{ll}4,4 & \mathrm{~g} \\
1,9 & \mathrm{~g}\end{array}$ & $\begin{array}{l}\text { toxische } \\
\text { Wirkung }\end{array}$ \\
\hline $\begin{array}{l}\text { 4. Versuch: } \\
\text { unbehandelt } \\
\text { Miracil D } \\
8 \times 50 \mathrm{sc} \text {. }\end{array}$ & $\begin{array}{l}14 \\
14\end{array}$ & $\begin{array}{l}1 \\
1\end{array}$ & $\begin{array}{l}2 \\
7\end{array}$ & $\begin{array}{r}11 \\
6\end{array}$ & $\begin{array}{ll}2,0 & \mathrm{~g} \\
1,2 & \mathrm{~g}\end{array}$ & \\
\hline $\begin{array}{l}\text { 5. Versuch: } \\
\text { unbehandelt } \\
\text { Miracil } \mathbf{D} \\
9 \times 25 \text { peroral }\end{array}$ & $\begin{array}{l}14 \\
14\end{array}$ & $=$ & $\stackrel{8}{8}$ & $\begin{array}{l}6 \\
6\end{array}$ & $\begin{array}{ll}5,8 & \mathrm{~g} \\
3,5 & \mathrm{~g}\end{array}$ & \\
\hline $\begin{array}{l}\text { 6. Versuch: } \\
\text { unbehandelt } \\
\text { Miracil D } \\
8 \times 20 \mathrm{sc} \text {. }\end{array}$ & $\begin{array}{l}14 \\
14\end{array}$ & $\begin{array}{l}1 \\
1\end{array}$ & $\bar{I}$ & $\begin{array}{l}13 \\
12\end{array}$ & $\begin{array}{ll}2,8 & \mathrm{~g} \\
2,9 & \mathrm{~g}\end{array}$ & $\begin{array}{l}\text { ohne } \\
\text { Wirkung }\end{array}$ \\
\hline $\begin{array}{l}\text { 7. Versuch: } \\
\text { unbehandelt } \\
\text { Miracil D } \\
6 . \times 10 \text { se. }\end{array}$ & $\begin{array}{l}14 \\
14\end{array}$ & $=$ & $\begin{array}{l}1 \\
1\end{array}$ & $\begin{array}{l}13 \\
13\end{array}$ & $\begin{array}{ll}2,3 & \mathrm{~g} \\
2,3 & \mathrm{~g}\end{array}$ & $\begin{array}{l}\text { ohne } \\
\text { Wirkung: }\end{array}$ \\
\hline
\end{tabular}

Benzpyrensarkom der Ratte.

Ratten erhielten $10 \mathrm{mg}$ Benzpyren in öliger Lösung subcutan. Nach 12 Wochen wurde die Behandlung begonnen, und zwar erhielten die Tiere der Versuchsgruppe in einigen Tagen Abstand im ganzen 9 Einzeldosen von je $50 \mathrm{mg} / \mathrm{kg}$ Miracil subcutan. Die Tumoren traten etwa von der 14 . Woche an auf, nach $4 \frac{1}{2}$ Monaten wurden alle Tiere getötet.

\begin{tabular}{l|l|l|l|l|r|}
\hline $\begin{array}{l}\text { 8. Versuch: } \\
\text { unbehandelt }\end{array}$ & 26 & 2 & 7 & 17 & $17,2 \mathrm{~g}$ \\
Miracil sc. & 26 & $\mathrm{i}$ & 6 & 19 & $7,4 \mathrm{~g}$ \\
$9 \times 50 \mathrm{mg} / \mathrm{kg}$ & & & & &
\end{tabular}

Bei parenteraler Anwendung in 1/2- bis 1\% iger Lösung bewirkt das Miracil D eine deutliche lokale Reizung. Die mehrmalige Verabreichung von $100 \mathrm{my} / \mathrm{kg}$ führt bei Mäusen und Ratten bei einem Teil der Tiere zum Tode. Dosen zwischen 25 und $100 \mathrm{mg}$ pro $\mathrm{kg}$ haben eine deutiche Hemmung des Tumorwarhstums zur Folge, während kleinere Dosen als $25 \mathrm{mg} / \mathrm{kg}$ in dieser Beziehung unwirksam sind.

Weitere Versuche mit verwandten Verbindungen werden noch durchgeführt.

Aus den Forschungslaboratorien der Farbenfabriken Bayer in Elberteld.

G. Hackmann, R. Gönnert und H. MauB.

Eingegangen am 20. Januar 1949.

1) Mau B, H., Chemische Berichte Jg. 81, S. 19 (1948)

2) Kikuth, W., Gönnert, K. und MauB, H., Naturwissenschaften 253 . Jg. 33 (1946).

3) Gönnert, R., Naturwissenschaften 347. Jg. 34 (1947).

s) Weyland, R., Dissertation Köln (1948). 